\title{
I know you are looking to me: Enabling eye-gaze communication between small children and parents with visual impairments
}

\author{
Mauro Avila Soto \\ Technical University of Ilmenau \\ Ilmenau, Germany
}

\author{
Najmeh Barzegar \\ Technical University of Ilmenau \\ Ilmenau, Germany \\ najmeh.barzegar@tu-ilmenau.de
}

\begin{abstract}
Eye-gaze interaction is a relevant mean of communication from the early infancy. The bonding between infants and their care-takers is Strengthened through eye contact. Parents with visual impairments are excluded of this type of interaction with their children. Thus, nowadays computer vision technologies allow to track eye-gaze with different purposes, even users with visual impairments are enable to recognize faces. This work starts from the following research question: Can current available eye tracking solutions aid parents with visual impairments to have eye-gaze interaction with their young infants children?

We devised a software prototype based on currently available eye tracking technologies which was tested with three sets of visually impaired parents and their young infant children to explore the possibility to assist those parents to have eye-gaze interaction with their children. The experience was documented as semi-structured interviews which were processed with a content analysis technique. The approach got positive feedback in the functionality and Emotional interaction aspects.
\end{abstract}

\section{KEYWORDS}

computer vision, visual impairment, Sensory replacement, social interaction

\section{ACM Reference Format:}

Mauro Avila Soto and Najmeh Barzegar. 2021. I know you are looking to me: Enabling eye-gaze communication between small children and parents with visual impairments. In 12th Augmented Human International Conference (AH2021), May 27-28, 2021, Geneva, Switzerland. ACM, New York, NY, USA, 4 pages. https://doi.org/10.1145/3460881.3460883

\section{INTRODUCTION}

Eye gaze signals play an important role in human communication. Interlocutors consciously or unconsciously uses gaze or eye contact to communicate with a conversation partner. These visual cues are frequently used by the sighted in social interactions $[2,9,10]$. This behavior is observable from early infanse, preview to the development of language ability [8]. However, those signals and cues are inaccessible for people with visual impairments (VI) [15].

This work is licensed under a Creative Commons Attribution International 4.0 License. AH2021, May 27-28, 2021, Geneva, Switzerland

(C) 2021 Copyright held by the owner/author(s).

ACM ISBN 978-1-4503-9030-9/21/05.

https://doi.org/10.1145/3460881.3460883
In young infants direct eye contact represents a first stage of social interaction, bonding, and basic communication with their care-takers[7]. When a parent presents a lebel of visual impairment which hinders or precludes eye-gaze interaction, the absenceof this kind of early non-verbal communication might result in a gap in the parenting experience for those visually impaired parents.

Computer vision technologies have generated several solutions to complement some aspects of person to person visual interaction which are difficult for users with visual impairments. Nowadays there are available different face recognition systems [3] to help users with visual impairments to recognize their friends, even embodying those solutions as wearable applications [13]. Some of those solutions are based on face recognition algorithms which provide recognition results in near real time with high accuracy [19]. In addition, computer vision algorithms can recognize people's facial expressions [18] and facial attributes such as eyes and facial hair [1]. These technologies can potentially enable users with VI to recognize their friends and better engage in social activities [14].

Although Eye tracking technologies are currently able to trace eye-gaze on a display, trigger the shutter of a camera, and other applications, computer aided eye-gaze detection within social interactions has gottten limited attention, even leeser in the context of computer vision assistive technology for users with VI. Considering the importance of direct eye contact before children shall be able to speak, $[4,16]$, and the difficulty of visually impaired parents to maintain this kind of non-verbal interaction with their young infants children, we defined the inquiry: Can current available eye tracking solutions aid parents with VI to have eye-gaze interaction with their young infants children?

In this work we report the implementation of a Eye gaze tracking tool which notifies users with visual impairments when an someone is looking to him or her. We deviced a prototype based on currently available Eye traking software deployed on standar computer hardware. This apparatus aimed to enable visually impaired parents to be aware when their baby children attempt to stablish direct eye-contact. Three test subject sets where recruited, three severely visually impaired parents (two fathers and a mother) and their three sighted babies. The tests were set up as Casual table top interactions among the babies and their parents with VI. Qualitative data was gathered through the analysis of semi-structured interviews conducted after the tests.

With this methodology we found evidence about the functionality and usability of the Eye gaze tracking tool in the context of the interaction between users with VI and young infants (yet unable to speak), conjointly we documented how enabling a rudimentary eyegaze interaction between sighted young infants and their parents 
with visual impairments has meant a positive emotional impact for those parents.

In the second section we describe the apparatus, in the third one we present our participants, the fourth section talks about the set up of the prototype, the fifth section describe the main test, and sixth part gives our conclusive ideas and outline further research work.

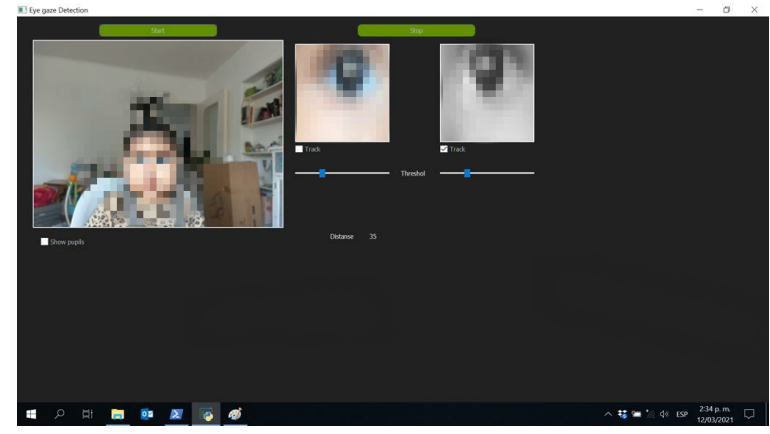

Figure 1: View of the EGT tool GUI. The eye-gaze of a infant subject is being detected (Due Details of confidentiality subject's face is blurred.)

\section{THE APPARATUS}

The prototype's first development stage should provide a reliable method to detect eye contact, thus we focused in Eye gaze tracking (EGT) techniques. An eye gaze tracker (EGT) is a device that computes the user's direction of gaze. If information about the objects in the scene is available, the point of regard can be computed as the intersection between the direction of gaze and the surface of the objects [12].

An existing EGT application writen in python was modified. [6]. This tool is able to track eye-gaze (it can differenciate between left and right eye) and highlight the eyes contour with visual indicators when eyes move at a configurable angle. For the purposes of this research, the code was modified with the deployment of a function which offer auditory feedback when it detects a direct eye-gaze in a concrete treshold of time and angle.

\subsection{EGT application features}

- It includes a GUI of 3 frames for displaying whole camera captured image, right eye and left eye. It has 3 check boxes to show pupils, tracking left eye, and right eye (see figure 1)

- It starts with turning the camera on and capturing the video and start detecting face and then following the eyes. After detection of right eye it is listened a message "right eye detected".

- after detecting left eye again it is listened the related message and when both eyes are detected it is listened "Left and right eyes detected".

- These messages will repeat in 7 th second and 15 th second as well. This feature aimed to define a eye contact threshold adapting the work of Farroni et al. [5] about eye-gaze perception during infancy.
- It has also the ability of detecting distance between user and camera and after detection of eyes it can be seen the distance on the screen as text and also it is listened the voice message which declare the distance.

in this way a EGT device able to detect direct eye-gaze can be deployed with the adequate hardware and software executing the modified python code. The integration of EGT software and the deployed hardware will be called EGT tool. Considering the condition of remote testing, participants utilized their own hardware to deploy the EGT tool.

\section{PARTICIPANTS}

To test this approach we looked for parents with visual impairments with young children (younger than one year old, or those children who are not able to speak yet). We recruited our participants through email lists dedicated to people with visual impairments. Three volunteers offered their participation. A mother of 34 years old from Spain with a baby of 8 months, a father of 35 years old from United States with a baby of 10 months, and a father of 32 years old from Spain with a 8 months baby. All the parents are within the group of severe visual impairment from early childhood, the babies present a normal development in their eye-sight.

Participants received by email a consent form which was read and accepted by children's legal tutors. All participants agreed to participate with the condition to keep anonymity of them and their children. Participants declared to be able to set up by their selves the corresponding Python environment and its required libraries.

\section{SETTING UP THE EGT TOOL}

To run the EGT software it is required Python version 3, in the case of participants setting, they installed Python 3.7, they are also required a set of libraries: Sip version 4.19.8, Beepy version 1.0.7, opencv-python version 4.2.0.34, numpy version 1.18.3, pyttsx3 version 2.87, PyQt5 version 5.14.2, and PyQt5-sip version 12.7.2.

recruited participants were instructed by email in the installation and setting of all the requirements to make run the EGT tool within certain parameters, once this was fulfilled, participants notified experimenters, then the conduction of a functionality based test [20] was requested seeking to observe and document any possible Functional peculiarities.

\subsection{Remote conducted EGT tool functionality test}

At first instance the test aimed to measure three functionality variables Effectiveness, Stability and Usability [17]., which are described as follows within the context of our participants and the objective of this experiment.

Effectiveness: This element is basically how succefully can the EGT tool fulfill the expected functions. This is in principle a simple reason success/failure between the times of compliance per number of tests.

Stability: This is a time measurement which indicates the time of response for each evaluated function; or if is it the case, how much time does the platform takes before crash down. 
Usability: This element shall be a measure of efficiency and satisfaction in the use of the EGT tool. In this case this should be in the context of the same functionality based test.

4.1.1 Reported results of the functionality based test. Our three participants reported as satisfactory the effectiveness and the stability, the only crashing down case reported was when the application stoped to work when it was executed and the default system camera was not detected. The EGT tool was able to detect direct eye-gaze at distances of $0.5,1$, and 1.5 meters, and when the observer was located in an arc with a range of 45 degrees at the left and the right from the focus center of the used camera. The tool was not able to detec eye-gaze if the observer wears glasses. The tool was not tested with any elevation or Slope angle. The distance estimate feature did not offered accuracy, giving an estimation error of around 50 centimeters. The software prototype was executed in laptop computers with Windows 10 Home as operating system, a processor Intel I5 and 16 gigabytes of RAM, and integrated GPUs, they have as system's default camera USB webcams with a sensor resolution of $4 \mathrm{~K}$.

Participants reported the tool as usable once it was properly arranged. The TTS notifications where understandable, however the arrangement using a computer and wired cameras was defined as cumbersome.

\section{EYE-GAZE DETECTION TEST}

The test were conducted between December 2020 and January 2021. Due contact Restriction Measures, supervision in person was not possible. Participants arranged the set up by their selves guided by instructions provided by email (see section 4 ).

The test setting was arranged as follows: (a) The parent and the child were located in a space where they shall be approximately at the same line of eye-gaze (e.g. the parend at the table and the child sitting on a baby chair). (b) The sensor of the camera is located close to the VI parent's face. (c) Parents start to interact between them and with the child. (d) VI parent listen to the notifications when the prototype detects the child looks to him or her. This Activity was conducted approximately during thirty minutes in three different dates selected under parents criterion.

Once the experiment was concluded participants conntacted the experimenter and a semi-structured interview was conducted online. The interview was based, but not constrained on the following questions:

- In daily life situations, are you aware when your child try to stablish eye contact with you?

- Do you consider relevant to be able to response to eye contact attempts from your child?

- How do you realize that your child request attention from you?

\subsection{Qualitative data analysis}

The three interviews were processed with an analysis methodology named Content Analysis which is a research tool used to determine the presence of certain words, themes, or concepts within some given qualitative data [11].
The content analysis method gave as output the following key statement concepts:

- I did not realize that my child looks at me so often.

- Even listening to noices and babbling I am not accurately aware when my baby looks at me.

- I feel more confident interacting with my child if I am able to response to eye contact.

- It is very helpful to be aware by my self when my child ask for attention specifically from me.

Within the participans depositions during the interviews, they provided positive feedback to the possibility to have a tool which can enhance their interaction with their children, we can quote individual comments. P1: When you deal with a small person unable to speak, and you can not see anything, you must guess half of times if he looks for your attention or aid. This stuff (EGT Tool) would be great when I play with my baby on the floor. P2: This device is excelent to integrate our baby in our conversations during lunch, I know he ccan not speak, but it is cool to be able to focus my face to him when I realize that he is looking at me. P3: I feel so nice when I listened my baby babbling and the computer said she was looking to me. Actually she was trying to talk to me.

\section{DISCUSSION, CONCLUSIONS, AND FUTURE WORK}

This work presents a very early stage for a research line which seeks to enable visually impaired users to participate in the non-verbal interaction possibilities of eye-gaze with their young infant children. We deviced and tested a prototype with three participant sets including visually impaired parents and their infants. We demonstrated our approach functionality and the potential impact which it can have in the daily-life interaction between parents with VI and their babies. It must be specified that the conduction of the tests was fulfilled in remote. Experimenters trusted in participants declarations about technical arrangements and tests carrying out.

From the conducted tests we can point out the following statements:

- An Eye gaze tracking tool can assist in a functional way parents with VI in their interaction with their infants leading to a positive answer to the research question addressed in section 1.

- Assistance in the eye-gaze interaction between parents with VI and their sighted infants can have a positive impact in the development of the parent-child relationship.

- Parents with VI report a improvement in their autonomy as care-takers through eye-gaze interaction aid.

The limited number of participants and the early stage of our prototype stablish requirements of further iterations seeking a quantifiable usability measure, nevertheless the collected quantitative data indicate the User Experience and interaction design alternatives to approach. Experimenters and participants agreed in a future work line aiming for a prototype which can provide feedback when the child look at different objects (e.g. hazardous objects like electrical outlets), and when the child does Facial expressions (e.g. Smiles, 
Expressions of disgust or fear). Haptic notifications can be implemented to enhance the interactivity with the device. The addition of machine learning features can enrich the experience through infants behavior prediction. The logical course of action leads to shrink the prototype into a mobile device, however it must be defined if a smartphone device offers suitable functionality.

\section{REFERENCES}

[1] N Bellustin, A Telnykh Kovalchuck, O Shemagina, V Yakho, Y Kalafati, Abhishek Vaish, Pinki Sharma, and Shirshu Verma. 2011. Instant human face attributes recognition system. Int. F. Adv. Comput. Sci. Appl., Special Issue Artif. Intel 3 (2011), 112-120.

[2] Ellen Berscheid. 1981. Silent Messages: Implicit Communication of Emotions and Attitudes. Psyccritiques 26, 8 (1981).

[3] Shonal Chaudhry and Rohitash Chandra. 2015. Design of a mobile face recognition system for visually impaired persons. arXiv preprint arXiv:1502.00756 (2015).

[4] Teresa Farroni, Mark H Johnson, Margaret Brockbank, and Francesca Simion 2000. Infants' use of gaze direction to cue attention: The importance of perceived motion. Visual cognition 7, 6 (2000), 705-718.

[5] Teresa Farroni, Mark H Johnson, and Gergely Csibra. 2004. Mechanisms of eye gaze perception during infancy. Fournal of cognitive neuroscience 16, 8 (2004), $1320-1326$.

[6] Stepan Filonov. 2019. Stepacool - Eye-Tracker. https://github.com/stepacool/EyeTracker.

[7] Tobias Grossmann, Mark H Johnson, Teresa Farroni, and Gergely Csibra. 2007 Social perception in the infant brain: gamma oscillatory activity in response to eye gaze. Social cognitive and affective neuroscience 2, 4 (2007), 284-291.

[8] Bahia Guellai and Arlette Streri. 2011. Cues for early social skills: direct gaze modulates newborns' recognition of talking faces. PloS one 6, 4 (2011), e18610.
[9] Adam Kendon. 1967. Some functions of gaze-direction in social interaction. Acta psychologica 26 (1967), 22-63.

[10] Mark L Knapp, Judith A Hall, and Terrence G Horgan. 2013. Nonverbal communication in human interaction. Cengage Learning.

[11] Klaus Krippendorff. 2018. Content analysis: An introduction to its methodology. Sage publications.

[12] Marcio RM Mimica and Carlos Hitoshi Morimoto. 2003. A computer vision framework for eye gaze tracking. In 16th Brazilian Symposium on Computer Graphics and Image Processing (SIBGRAPI 2003). IEEE, 406-412.

[13] Laurindo de Sousa Britto Neto, Vanessa Regina Margareth Lima Maike, Fernando Luiz Koch, Maria Cecília Calani Baranauskas, Anderson de Rezende Rocha, and Siome Klein Goldenstein. 2015. A wearable face recognition system built into a smartwatch and the blind and low vision users. In International Conference on Enterprise Information Systems. Springer, 515-528.

[14] Sethuraman Panchanathan, John Black, Mike Rush, and Vivek Iyer. 2003. iCare-a user centric approach to the development of assistive devices for the blind and visually impaired. In Proceedings. 15th IEEE International Conference on Tools with Artificial Intelligence. IEEE, 641-648.

[15] Shi Qiu, Jun Hu, and Matthias Rauterberg. 2015. Nonverbal signals for faceto-face communication between the blind and the sighted. In Proceedings of International Conference on Enabling Access for Persons with Visual Impairment. 157-165.

[16] Silvia Rigato, Enrica Menon, Mark H Johnson, Dino Faraguna, and Teresa Farroni. 2011. Direct gaze may modulate face recognition in newborns. Infant and Child Development 20, 1 (2011), 20-34.

[17] Suzanne Robertson and James Robertson. 2012. Mastering the requirements process: Getting requirements right. Addison-wesley.

[18] Caifeng Shan, Shaogang Gong, and Peter W McOwan. 2009. Facial expression recognition based on local binary patterns: A comprehensive study. Image and vision Computing 27, 6 (2009), 803-816.

[19] Yaniv Taigman, Ming Yang, Marc'Aurelio Ranzato, and Lior Wolf. 2014. Deepface: Closing the gap to human-level performance in face verification. In Proceedings of the IEEE conference on computer vision and pattern recognition. 1701-1708.

[20] Karl Wiegers and Joy Beatty. 2013. Software requirements. Pearson Education. 